\title{
Effects of early-life exposure to topsoil on the muscle fiber characteristics and gene expression of weaned piglets
}

\section{Yongjie Wang}

University of Arkansas at Fayetteville

Tsung Cheng Tsai

University of Arkansas at Fayetteville

\section{Palika Morse}

University of Arkansas at Fayetteville

Shilei Zhang

University of Arkansas at Fayetteville

Charles Maxwell

University of Arkansas at Fayetteville

Jiangchao Zhao

University of Arkansas at Fayetteville

Yan Huang ( $\nabla$ yxh010@uark.edu )

University of Arkansas at Fayetteville

\section{Research Article}

Keywords: soil, group, expression, normal, muscle, significantly, fiber, antibacterial

Posted Date: August 4th, 2021

DOI: https://doi.org/10.21203/rs.3.rs-544070/v2

License: (c) (i) This work is licensed under a Creative Commons Attribution 4.0 International License.

Read Full License 
1 Effects of early-life exposure to topsoil on the muscle fiber characteristics and

\section{gene expression of weaned piglets}

3 Yongjie Wang ${ }^{1}$, Tsung Cheng Tsai ${ }^{1}$, Palika Morse ${ }^{1}$, Shilei Zhang ${ }^{1}$, Charles Maxwell ${ }^{1}$, Jiangchao

$4 \mathrm{Zhao}^{1}$, and Yan Huang ${ }^{*}$

$5{ }^{1}$ Department of Animal Science, Division of Agriculture, University of Arkansas, Fayetteville,

$6 \quad$ AR 72701, USA

$7 \quad$ * Correspondence to yxh010@uark.edu

8

9 Abstract

10 The objective of this experiment was to investigate the influence of early exposure to topsoil on

11 the muscle fiber characteristics and transcription related myogenesis, intramuscular fat

12 metabolism, muscle fiber types, and mTOR signaling pathway of weaned pigs. A Total of 180

13 piglets were separately assigned to No soil, Antibacterial soil, and Normal soil group (each

14 group, $n=60$ ), and were fed ad libitum with common antibiotic-free corn-soybean meal diets until

15 day-31. Ten pigs from each group with similar body weight were selected to be slaughtered, and

16 the longissimus dorsi (LD) muscle samples were collected for histological analysis and

17 measurements of genes and proteins expression levels. In the present study, the muscle fiber

18 diameter and the area of Normal soil and Antibacterial soil group were significantly higher than

19 No soil group $(P<0.05)$. The Normal soil significantly upregulated the gene expression of

20 MyoG compared to No soil and Antibacterial soil groups $(P<0.05)$. The gene expression of

21 CD36 and CPT-1 of Normal soil group was significantly lower than No soil group $(P<0.05)$,

22 while HSL expression of Normal soil group was significantly higher than Antibacterial and No 
23 soil groups $(P<0.05)$. The MyHC I of Normal soil group was significantly higher than No soil

24 group $(P<0.05)$, but the expression MyHC IIa was lower than No soil group $(P<0.05)$. The

25 protein expression expressed the similar result with gene expression. In addition, the Normal soil

26 significantly increased the AMPK and mTOR phosphorylation compared to No soil and

27 Antibacterial soil groups $(P<0.05)$. These data suggest that early exposure to topsoil regulates

28 the muscle fiber growth, modulates the expression pattern related to myogenesis, muscle fiber

29 type, intramuscular fat metabolism, and increases the phosphorylation of mTOR and AMPK

30 pathways.

\section{Introduction}

33 In the modern pig industry, fewer than $6 \%$ of the pig raised in the United States are housed in

34 pasture of dirt pens, and most of them have been moved from outdoors into the slatted-floor

35 indoor systems ${ }^{1}$. This phenomenon was caused by the increased space requirements as well as a

36 need for more complex management practices for outdoor systems compared to indoor systems ${ }^{2}$.

37 The pigs fed indoor rarely have the opportunity to touch the soil, which is a complex system

38 consists of air, water, minerals, organic matter, and biota ${ }^{3}$. The effects of this environmental

39 change are still understudied for the fact that the outdoor system is not a feasible means of swine

40 production for the majority pig industry. However, previous studies show that feeding piglets

41 outdoors during lactation improves growth performance, carcass weight, and feed efficiency ${ }^{4,5}$.

42 In addition, it was reported that early exposure to agricultural soil modulates the gut microbiota

43 maturation of pigs during their early life ${ }^{6}$. Despite these discoveries, many questions remain in 
44 regard to the roles of the exposure to outdoor soil in regulating myogenesis, muscle fiber type,

45 intramuscular fat metabolism.

46 A previous investigation confirmed that the muscle fiber characteristics could be affected by the

47 animal rearing environment ${ }^{7}$. The muscle fiber characteristics (diameter, number, area) and

48 fiber type composition are closely related to each other ${ }^{8}$, and it is a reflection of animal growth

49 rate ${ }^{9}$. Usually, muscle fiber types are defined by the isoforms of myosin heavy chain (MHC) as

50 MyHC I, IIa, IIb, and IIx, based on their different ATPase types ${ }^{10}$. The gene expression related

51 to the intramuscular fat metabolism, such as PPAR $\gamma, \mathrm{CPT} 1, \mathrm{SREBP} 1$, etc., was affected by

52 rearing systems ${ }^{11}$. Different rearing environments may also impact the lipid, protein, and energy

53 metabolism in skeletal muscle by modulating the mechanistic target of the rapamycin (mTOR)

54 signaling pathway ${ }^{12}$.

55 The objective of this experiment was to explore the effects of exposure to topsoil during pre-

56 weaning on the muscle fiber characteristics and expression patterns of genes and proteins related

57 to myogenesis, muscle fiber type, intramuscular fat metabolism, and mTOR pathway.

59 Results

60 Muscle fiber characteristics

61 The histochemical section of LD muscle is shown in Figure 1. The muscle fiber characteristics

62 consisted of muscle fiber diameter, fiber area, and the number of fibers are shown in Table 2.

63 The muscle fiber diameter of Antibacterial soil group was significantly higher than Normal and

64 No soil groups $(P<0.05)$, and the Normal soil group had a significantly higher diameter than the 
65 No soil group $(P<0.05)$. The muscle fiber area of Antibacterial soil and Normal soil groups was

66 significantly greater than No soil group $(P<0.05)$. However, there were no significant

67 differences in muscle fiber number among each group $(P>0.05)$.

68

69 Myogenesis relative gene expression

70 The gene expression related to myogenesis (MyoG, Myf5, and MSTN) in the LD muscle was

71 measured by RT-PCR is presented in Figure 2. The Normal soil significantly upregulated the

72 gene expression of MyoG of LD muscle compared to No soil and Antibacterial soil groups $(P<$

73 0.05). However, there was no significant gene expression differences of Myf5 and MSTN

74 between each group $(P>0.05)$.

\section{Intramuscular fat relative gene expression}

77 The gene expression related with intramuscular fat deposition and lipolysis are shown in Figure

783 and Figure 4. For fat deposition, the gene expression of CD36 in No soil group was

79 significantly higher than Normal soil group $(P<0.05)$, but there was no significant difference

80 between No soil group and Antibacterial soil group $(P>0.05)$. There were no significant

81 differences of gene expression of PPAR $\gamma$, FAS, and SREBP-1 between each group $(P>0.05)$.

82 The gene expression related with lipolysis such as HSL was significantly upregulated in Normal

83 soil group compared to Antibacterial soil and No soil group $(P<0.05)$. The LPL expression of

84 Normal soil group was significantly higher than No soil group $(P<0.05)$, yet no significant

85 difference compared with Antibacterial soil group $(P>0.05)$ was noticed. However, the CPT1 
was down regulated in Normal soil group compared with No soil group $(P<0.05)$. Additionally, there was no significant difference of ATGL expression between each group $(P>0.05)$.

\section{Muscle fiber type relative gene expression}

The expression levels of MyHC I, MyHC IIa, MyHC IIb and MyHC IIx were illustrated in

Figure 5. The MyHC I of Normal soil group was significantly higher than No soil group $(P<$ 0.05), but no significant difference compared to Antibacterial soil group $(P>0.05)$. However, the MyHC IIa of Normal soil group was significantly down regulated compared with No soil group $(P<0.05)$. There were no significant differences of MyHC IIb and IIx between each group $(P>0.05)$.

\section{Protein expression related with intramuscular fat and myogenesis}

HSL, PPAR $\gamma$, Myf5, and MSTN expressions were further measured using Western blot, and the results were displayed in Figure 6. The protein abundance of HSL in Normal soil group was significantly higher than Antibacterial soil and No soil group $(P<0.05)$. In addition, PPAR $\gamma$ and MSTN protein abundances were significantly decreased in Normal soil group compared with the other two groups $(P<0.05)$. These protein expressions were consistent with the PCR results. However, there was no significant difference of Mfy5 abundance between each group $(P>0.05)$. 
106 The protein abundances of MyHC I, MyHC IIa, MyHC IIb and MyHC IIx were determined via

107 Western blot (Figure 7). The protein expression of MyHC I and MyHC IIa was comparable with

108 the PCR results. The MyHC I of Normal soil group was significantly greater than No soil and

109 Antibacterial soil group $(P<0.05)$, and the MyHC IIa of Normal soil group was significantly

110 lessened $(P<0.05)$. The MyHC IIx protein abundance in Normal soil group was also

111 significantly decreased compared with other two groups $(P<0.05)$. Consistently, the MyHC IIb

112 protein abundance was not significantly different among all groups $(P>0.05)$.

\section{Protein expression related with mTOR pathway}

115 AMPK and mTOR signaling pathways were determined using Western blotting analysis, and the

116 outcomes were demonstrated in Figure 8. The results showed that the Normal soil significantly

117 increased the AMPK and mTOR phosphorylation compared to No soil and Antibacterial soil

118 groups $(P<0.05)$, and no significant differences were obvious between No soil and Antibacterial

119 soil groups $(P>0.05)$.

\section{Discussion}

122 In relation to previous studies, muscle fiber characteristics (muscle fiber diameter, number, cross

123 section area, and muscle fiber type) were affected by intrinsic factors (breed, sex, and age) and

124 extrinsic factors such as nutrition value ${ }^{13-15}$. Based on the results, our study indicated that

125 different topsoil treatments also had effects on the muscle fiber characteristics and the

126 transcription related with myogenesis and muscle fiber type. Larzul, et al. ${ }^{9}$ pointed out that the

127 muscle fiber cross section area had a positive relationship with the pig growth rate. Relevantly, 
128 the muscle fiber area of Normal soil group and Antibacterial soil group was significantly higher

129 than No soil group and indicated a better muscle growth rate. In addition, the muscle fiber

130 diameter is positively related to the cross-section area ${ }^{13}$, and our results were consistent with this

131 theory.

132 Hyperplasia (increasing muscle fiber number) and hypertrophy (increased fiber size) are two

133 main processes that regulate muscle growth ${ }^{16}$. MRFs are a family of helix-loop-helix

134 transcription aspects, including MyoG, Myf5, etc., that modify muscle hyperplasia and

135 hypertrophy ${ }^{17}$. Myf5 is the primary MRFs which directly proliferates myogenic progenitor cells

136 towards a myogenic lineage, and MyoG is the secondary MRFs which regulates the

137 differentiation and fusion of myoblasts to form myofibers ${ }^{18,19}$. The expression of MSTN is

138 mainly in skeletal muscle and its expression has a negative relationship with muscle growth ${ }^{20}$. It

139 was also reported that the mutation of MSTN increased the muscle growth and muscle mass in

140 many animals including pigs, sheep, rabbits, and cattle ${ }^{21-24}$. This experiment confirmed that

141 exposure to topsoil during the pre-weaning stage changed the transcription related with

142 myogenesis. Exposure to Normal soil upregulated the gene expression of Myf5, while

143 downregulated the MSTN transporter. The results indicated that exposure to topsoil had a

144 positive impact on the myogenesis of piglets.

145 According to our results, the transcription of different muscle fiber types of weaned pigs was

146 also affected by the topsoil. In general, there are four different muscle fiber types (type I, type

147 IIa, type IIb, and type IIx) which can be detected in pig skeletal muscle, and they are

148 distinguished by different types of myosin heavy chain (MyHC I, IIa, IIb, and IIx) ${ }^{10}$. Type I is

149 slow-oxidative fiber, type IIa is fast oxide-glycolytic fiber, and both type IIb and IIx are fast

150 glycolytic fibers ${ }^{25}$. Different muscle fiber types represent different ATPase characteristics of 
151 fibers ${ }^{26}$, for example, type I fiber is rich in mitochondria which provides ATP by its fatty acid 152 oxidation, and type II fibers, which are classified as IIa, IIb, or IIx by its myosin heavy chain 153 (MyHC) isoforms expression, utilize glucose to supply energy ${ }^{10,27}$. The muscle fiber types are 154 impacted by complicated intrinsic and extrinsic factors such as breed, gender, age, nutrient level, 155 and physical activity ${ }^{28}$. Based on this theory and our transcription results, exposure to the 156 topsoil, as an extrinsic factor, also altered the muscle fiber type composition and the energy 157 utilization forms of weaned pigs, and contacting with Normal soil increased the proportion of 158 type I fiber which relies on the oxidative activity while decreased the type II fibers which have 159 more glycolytic metabolism. The changes of muscle fiber characteristics of weaned pigs may 160 also affect the postmortem metabolism or even the consequential meat quality.

161 In addition, the gene and protein expression related with intramuscular fat deposition and fat 162 removal ofpiglets were influenced by the exposure to topsoil. For the intramuscular fat 163 deposition, exposure to Normal soil downregulated the CD36 gene and PPAR $\gamma$ protein 164 expression. CD36 is a primary fatty acid transporter expressed in animal skeletal muscle ${ }^{29,30}$, 165 and its abundance on the plasma membrane of obesity animals has a positive correlation with the 166 rate of fatty acid uptake ${ }^{31}$. PPAR $\gamma$ is a ligand-activated transcription factor, expressed in many 167 tissues (skeletal muscle and adipocytes) which accelerates adipogenesis and insulin sensitivity $168 \quad 32,33$, and its expression regulates the stimulation of adipocyte differentiation and fat deposition

$169 \quad{ }^{34}$. In terms of lipolysis, Normal soil unregulated the HSL, LPL expression, while downregulated 170 the CPT1 gene expression. HSL detached fatty acids from intracellular triacylglycerol for 171 oxidation and exportation ${ }^{35}$. LPL is a rate-limiting enzyme for the hydrolysis of triacylglycerol, 172 and its catalyzed reaction products, fatty and monoacylglycerol, are used by adipose tissue and 
173 skeletal muscle as element of neutral lipids ${ }^{36}$. CPT1 was reported to be correlated with

174 mitochondrial fatty acid oxidation ${ }^{37}$.

175 The mTOR pathway plays an important role in modulating amino acid metabolism and reflecting

176 the availability of amino acid ${ }^{38,39}$. In addition, AMPK is a key energy sensor which regulates the

177 cellular energy ${ }^{40}$, and it can also modulates oxidative stress and mitochondrial function ${ }^{41}$. In

178 this investigation, we found that exposure to topsoil increased the mTOR and AMPK signaling

179 pathway in weaned pigs, and it activated their phosphorylation. The results indicated that the

180 amino acid and cellular energy metabolism of piglets were modulated by the exposure to topsoil

181 during weaning period.

182 The current experiment proved that exposure to topsoil promotes the myogenesis, modulates the

183 transcription related with muscle fiber types, intramuscular fat deposition and lipolysis, regulates

184 the AMPK and mTOR signaling pathway of weaned piglets.

\section{Material and methods}

\section{Animals and experimental design}

188 The University of Arkansas's Institutional Animal Care and Use Committee approved all

189 experimental procedures involving animals during the study (ethical approval code: 18059). This

190 study followed the Animal Research: Reporting of In Vivo Experiments (ARRIVE) guidelines

191 (PLoS Bio 8(6), e1000412,2010). All experiments were performed in accordance with relevant

192 guidelines and regulations. All 30 sows used in the study were blocked by parity and farrowing

193 body weight. Piglets from each litter were cross-fostered within 24 hours across three sows

194 (within similar parity and body weight). Six piglets with similar body weight from each litter 
$195(\mathrm{n}=180)$ were individually transferred to the same pen in the nursery facility, and kept in their

196 littermates for the entire trial. Piglets were equally allocated to the No soil group, Antibacterial

197 soil group, and Normal soil group (60 piglets in each group). No soil group was exposed to an

198 empty pan, Antibacterial soil group was exposed to a pan with $1 \mathrm{~kg}$ of irradiated topsoil

199 (Sterigenics, Fort Worth, TX) to kill bacteria in the soil, and Normal soil group was exposed to a

200 pan with $1 \mathrm{~kg}$ of topsoil (Sod Store, Inc., Tontitown, AR). They were fed ad libitum with

201 common antibiotic-free corn-soybean meal diets in littermates for 31 days.

\section{Animal slaughter and sampling}

203 The day prior to harvest, all pigs were weighed, and the pigs of median weight form each pen (10 204 piglets per group) were selected for sampling on day 31 . Following a $12 \mathrm{~h}$ period of fasting prior 205 to slaughter with the access of water, the pigs were transported to the University of Arkansas red

206 meat abattoir. Piglets were euthanized by a captive bolt and immediately followed by

207 exsanguination. Then, the muscle samples from longissimus dorsi (LD) of left carcass were

208 removed and subsequently snap frozen at $-80^{\circ} \mathrm{C}$ in liquid nitrogen for RNA isolation and protein

209 extraction process. Another piece of LD muscle from each pig was cut into $0.5 \times 0.5 \times 1.0 \mathrm{~cm}$

210 cube, and immediately fixed in $10 \%$ buffered neutral formalin solution for the histological

211 experiment.

\section{Histological analysis}

213 The LD muscle samples, fixed in $10 \%$ buffered neutral formalin solution, were dehydrated in

214 alcohol, cleared in xylene, infiltrated, embedded in paraffin ${ }^{42}$, and then were cut in to $3 \mu \mathrm{m}$

215 thickness. H\&E (hematoxylin and eosin) staining was used to treat the thickness for histological

216 study ${ }^{43}$. Stained cross-sections were viewed and photographed at $175 \times$ by $\mathrm{ZOE}^{\mathrm{TM}}$ Fluorescent 
217 Cell Imager (Bio-rad, Hercules, CA, USA). Five photographs of each cross-section of LD

218 samples were taken, and then they were analyzed using Image-J software (National Institutes of

219 Health, Bethesda, MD, USA). The average muscle fiber numbers per area were obtained by

220 counting the total number of fibers in five areas $\left(700,000 \mu \mathrm{m}^{2}\right.$ for each area). The muscle fiber

221 diameter $(\mu \mathrm{m})$ and area $\left(\mu \mathrm{m}^{2}\right)$ of each LD muscle was measured by using 300 fibers in five

222 cross-sections of each sample.

\section{RNA isolation and cDNA synthesis}

224 The samples, stored in liquid nitrogen, were homogenized using the Precellys Evolution

225 homogenizer (Bertin Technologies, Rockville, MD, USA) with TRIzol ${ }^{\mathrm{TM}}$ Reagent (Thermo

226 Fisher Scientific, Cat. No. 15596026). After homogenization, Direct-Zol ${ }^{\mathrm{TM}}$ RNA Miniprep Kit

227 (Zymo Research, Cat. No. R2072) was used to extract the total RNA from each samples of LD

228 muscle. To eliminate genomic DNA contamination, resultant total RNA was then treated with

229 DNase I, RNase-free (Promega, Madison, WI, USA), in accordance with manufacturer's

230 instructions. The RNA concentration was assessed by NanoDrop (Agilent Technologies, Santa

231 Clara, CA, USA). Finally, the purified RNA samples were subjected to reverse-transcription

232 using iScriptTM Cdna Synthesis Kit (Bio-rad, Cat. No. 1708890) following the instructions

233 given.

\section{Real-time qPCR}

235 According to the recorded sequences showed in GenBank, the primers of $\beta$-actin, MyoG, Myf5,

236 MSTN, PPAR- $\gamma$, FAS, SREBP-1, CD36, CPT-1, ATGL, LPL, HSL, MyHC I, MyHC IIa, MyHC

237 IIb, MyHC IIx were designed using GenBank for pig genes (Table 1). $\beta$-actin was chosen as the

238 house-keeping gene to normalize target gene levels. Real-time qPCR was performed by using 
$\mathrm{iQ}^{\mathrm{TM}} \mathrm{SYBR}^{\circledR}$ Green Supermix (Bio-rad, Cat. No. 1708890) along with the manufacturer's instructions. The $2^{-\Delta \Delta \mathrm{Ct}}$ method was used to analyze the relative expression, and the relative expression was normalized and expressed as a ratio to the expression in the No soil group.

\section{Western Blot}

Total proteins of LD muscle samples were extracted using T-PER ${ }^{\mathrm{TM}}$ Tissue Protein Extraction Reagent (Thermo Fisher Scientific, Cat. No. 78510) according to the guidelines. The concentration of protein samples was measured by Micro $\mathrm{BCA}^{\mathrm{TM}}$ Protein Assay Kit (Thermo Fisher Scientific, Cat. No. 23235). Protein samples were separated on 7.5\% Mini-PROTEAN ${ }^{\circledR}$ $\mathrm{TGX}^{\mathrm{TM}}$ Precast Protein Gels, 15-well, $15 \mu \mathrm{l}$ (Bio-rad, Cat. No. 4561026), and then transferred onto Trans-Blot Turbo Midi Nitrocellulose Transfer Packs (Bio-rad, Cat. No. 1704159). Nonspecific antibodies were excluded by blocking the membrane with 5\% BSA (Bovine Serum Albumin) as blocking buffer for $120 \mathrm{~min}$ at $25^{\circ} \mathrm{C}$. The membranes were incubated with primary antibodies over night at $4{ }^{\circ} \mathrm{C}$ after blocking, and then were rinsed and incubated with secondary antibodies for $60 \mathrm{~min}$ at $25^{\circ} \mathrm{C}$. The antibodies, including PPAR $\gamma, \mathrm{HSL}, \mathrm{Myf5}, \mathrm{MSTN}, \mathrm{MyHC}$, MyHC IIa, MyHC IIb, MyHC IIx, p-AMPK, AMPK, p-mTOR, mTOR, GAPDH and HRPconjugated anti-rabbit Ab were purchased from ABclonal Technology Inc. (Woburn, MA, USA). The Clarity ${ }^{\mathrm{TM}}$ Western ECL Substrate (Bio-rad, Cat. No. 1705060) was used to visualize bands, and then the bands were detected by ChemiDoc ${ }^{\mathrm{TM}}$ Touch Gel Imaging System (Bio-rad, Hercules, CA, USA). The density of bands was normalized according to GAPDH content. The expression levels of different proteins were quantified using Image Lab Software (Bio-rad, Hercules, CA, USA) ${ }^{44}$. 


\section{Statistical analysis}

262 All data were analyzed by one-way ANOVA with SPSS 22.0 statistical software (SPSS Inc.,

263 Chicago, IL, USA). The results were expressed as the Mean $\pm \mathrm{SEM}$, and a $P<0.05$ was used to

264 determine statistical significance.

\section{References}

269

270

271

272

273

274

275

1 Bush, E. J. Swine 2000, Part Ii: Reference Of Swine Health And Health Management In The United States, 2000. (2002).

2 Dourmad, J.-Y. et al. Evaluating environmental impacts of contrasting pig farming systems with life cycle assessment. 8, 2027-2037 (2014).

3 Sing, C., Sing, D. J. E. o. s. s. T. \& Fromes, N. Y. Soil and human health. (2008).

4 Colson, V., Martin, E., Orgeur, P., Prunier, A. J. P. \& behavior. Influence of housing and social changes on growth, behaviour and cortisol in piglets at weaning. 107, 59-64 (2012).

5 Cox, L. \& Cooper, J. J. A. S. Observations on the pre-and post-weaning behaviour of piglets reared in commercial indoor and outdoor environments. 72, 75-86 (2001).

6 Vo, N., Tsai, T. C., Maxwell, C. \& Carbonero, F. J. A. Early exposure to agricultural soil accelerates the maturation of the early-life pig gut microbiota. 45, 31-39 (2017).

7 Gentry, J., McGlone, J., Miller, M. \& Blanton Jr, J. J. J. o. A. S. Environmental effects on pig performance, meat quality, and muscle characteristics. 82, 209-217 (2004).

8 Ryu, Y., Rhee, M.-S. \& Kim, B.-C. J. A.-a. j. o. a. s. Estimation of correlation coefficients between histological parameters and carcass traits of pig longissimus dorsi muscle. $17,428-433$ (2004).

9 Larzul, C. et al. Phenotypic and genetic parameters for longissimus muscle fiber characteristics in relation to growth, carcass, and meat quality traits in large white pigs. 75, 3126-3137 (1997).

10 Pette, D., Staron, R. S. J. M. r. \& technique. Myosin isoforms, muscle fiber types, and transitions. 50, 500-509 (2000).

11 Guo, Y. et al. Effects of different rearing systems on intramuscular fat content, fatty acid composition, and lipid metabolism-related genes expression in breast and thigh muscles of Nonghua ducks. 99, 4832-4844 (2020).

12 Lallès, J. \& Guillou, D. Pig intestine, weaning and dietary interventions. (Wageningen Academic Publishers, Wageningen, The Netherlands, 2015).

13 Miller, L., Garwood, V. \& Judge, M. J. J. o. A. S. Factors affecting porcine muscle fiber type, diameter and number. 41, 66-77 (1975).

14 Dwyer, C., Fletcher, J. \& Stickland, N. J. J. o. A. S. Muscle cellularity and postnatal growth in the pig. 71, 3339-3343 (1993). 
Dwyer, C. M., Stickland, N. C. \& Fletcher, J. M. J. J. o. A. S. The influence of maternal nutrition on muscle fiber number development in the porcine fetus and on subsequent postnatal growth. 72, 911-917 (1994).

16 Zhu, K. et al. Characterization of muscle morphology and satellite cells, and expression of muscle-related genes in skeletal muscle of juvenile and adult Megalobrama amblycephala. 64, 66-75 (2014).

17 Zammit, P. S. in Seminars in cell \& developmental biology. 19-32 (Elsevier).

18 Conboy, I. M. \& Rando, T. A. J. D. c. The regulation of Notch signaling controls satellite cell activation and cell fate determination in postnatal myogenesis. 3, 397-409 (2002).

19 Dhawan, J. \& Rando, T. A. J. T. i. c. b. Stem cells in postnatal myogenesis: molecular mechanisms of satellite cell quiescence, activation and replenishment. 15, 666-673 (2005).

20 McPherron, A. C., Lawler, A. M. \& Lee, S.-J. J. N. Regulation of skeletal muscle mass in mice by a new TGF-p superfamily member. 387, 83-90 (1997).

21 Grobet, L. et al. A deletion in the bovine myostatin gene causes the double-muscled phenotype in cattle. 17, 71-74 (1997).

$22 \mathrm{Bi}, \mathrm{Y}$. et al. Isozygous and selectable marker-free MSTN knockout cloned pigs generated by the combined use of CRISPR/Cas9 and Cre/LoxP. 6, 1-12 (2016).

23 Wang, X. et al. Multiplex gene editing via CRISPR/Cas9 exhibits desirable muscle hypertrophy without detectable off-target effects in sheep. 6, 1-11 (2016).

24 Lv, Q. et al. Efficient generation of myostatin gene mutated rabbit by CRISPR/Cas9. 6, 1-8 (2016).

25 Zierath, J. R. \& Hawley, J. A. J. P. B. Skeletal muscle fiber type: influence on contractile and metabolic properties. 2, e348 (2004).

26 Kushmerick, M. J., Moerland, T. S. \& Wiseman, R. W. J. P. o. t. N. A. o. S. Mammalian skeletal muscle fibers distinguished by contents of phosphocreatine, ATP, and Pi. 89, 7521-7525 (1992).

27 Schiaffino, S. \& Reggiani, C. J. P. r. Fiber types in mammalian skeletal muscles. 91, 1447-1531 (2011).

28 Lee, S., Joo, S. \& Ryu, Y. J. M. S. Skeletal muscle fiber type and myofibrillar proteins in relation to meat quality. 86, 166-170 (2010).

29 Bonen, A., Luiken, J. J., Arumugam, Y., Glatz, J. F. \& Tandon, N. N. J. J. o. B. C. Acute regulation of fatty acid uptake involves the cellular redistribution of fatty acid translocase. $\mathbf{2 7 5}$, 14501-14508 (2000).

30 Ibrahimi, A., Abumrad, N. A. J. C. O. i. C. N. \& Care, M. Role of CD36 in membrane transport of long-chain fatty acids. 5, 139-145 (2002).

31 Aguer, C. et al. Intramyocellular lipid accumulation is associated with permanent relocation ex vivo and in vitro of fatty acid translocase (FAT)/CD36 in obese patients. 53, 1151-1163 (2010).

32 Matsusue, K., Peters, J. M. \& Gonzalez, F. J. J. T. F. J. PPAR $\beta / \delta$ potentiates PPAR $\gamma$-stimulated adipocyte differentiation. 18, 1477-1479 (2004).

33 Hevener, A. L. et al. Macrophage PPAR $\gamma$ is required for normal skeletal muscle and hepatic insulin sensitivity and full antidiabetic effects of thiazolidinediones. 117, 1658-1669 (2007).

34 Janani, C., Kumari, B. R. J. D., Research, M. S. C. \& Reviews. PPAR gamma gene-a review. 9, 46-50 (2015).

35 Dolinsky, V. et al. Triacylglycerol hydrolase: role in intracellular lipid metabolism. 61, 16331651 (2004).

36 Wang, H., Eckel, R. H. J. A. J. o. P.-E. \& Metabolism. Lipoprotein lipase: from gene to obesity. 297, E271-E288 (2009).

37 Sebastián, D. et al. Novel role of FATP1 in mitochondrial fatty acid oxidation in skeletal muscle cells. 50, 1789-1799 (2009).

38 Jewell, J. L., Russell, R. C. \& Guan, K.-L. J. N. r. M. c. b. Amino acid signalling upstream of mTOR. 14, 133-139 (2013). 
39 Han, C. et al. Insulin stimulates goose liver cell growth by activating PI3K-AKT-mTOR signal pathway. 38, 558-570 (2016).

40 Hardie, D. G., Ross, F. A. \& Hawley, S. A. J. N. r. M. c. b. AMPK: a nutrient and energy sensor that maintains energy homeostasis. 13, 251-262 (2012).

$41 \mathrm{Wu}, \mathrm{W}$. et al. Cathelicidin-WA attenuates LPS-induced inflammation and redox imbalance through activation of AMPK signaling. 129, 338-353 (2018).

42 Meng, K. et al. Terahertz pulsed spectroscopy of paraffin-embedded brain glioma. 19, 077001 (2014).

43 Fischer, A. H., Jacobson, K. A., Rose, J. \& Zeller, R. J. C. s. h. p. Hematoxylin and eosin staining of tissue and cell sections. 2008, pdb. prot4986 (2008).

44 Herrero, L. et al. Alteration of the malonyl-CoA/carnitine palmitoyltransferase I interaction in the $\beta$-cell impairs glucose-induced insulin secretion. 54, 462-471 (2005).

\section{Acknowledgements}

\section{Author information}

Affiliations

Department of Animal Science, Division of Agriculture, University of Arkansas, Fayetteville, AR 72701, USA

Yongjie Wang, Tsung-Cheng Tsai, Shilei Zhang, Jiangchao Zhao, Yan Huang

\section{Contributions}

Y.H., T.T., C.M., and J.Z. conceived and designed the experiment; Y.W., P.M., and S.Z.

performed the experiments; Y.W. analyzed the data; Y.W. and Y.H. wrote and revised the paper. All authors have read and approved the final version of the manuscript. 
375 Correspondence to Yan Huang

376 Competing interests

377 The authors claim no competing interestes

378

379

380

381

382 Abbreviations

383 AMPK: AMP-activated protein kinase

384 ATGL: adipose triglyceride lipase

385 CD36: cluster of differentiation molecule

386 CPT-1: carnitine palmitoyltransferase 1

387 FAS: Fas cell surface death receptor

388 H\&E: hematoxylin and eosin

389 HSL: hormone-sensitive lipase

390 LD: longissimus dorsi

391 LPL: lipoprotein lipase

392 mTOR: mammalian target of rapamycin

393 MSTN: myostatin

394 MyHC-I: myosin heavy chain I

395 MyHC-IIa: myosin heavy chain IIa 
396 MyHC-IIb: myosin heavy chain IIb

397 MyHC-IIx: myosin heavy chain IIx

398 Myf5: myogenic factor 5

399 MyoG: myogenin

400 PPAR- $\gamma$ : peroxisome proliferator activated receptor gamma

401 SREBP-1: sterol regulatory element binding transcription factor 1

402

403

$404 \quad$ (A)

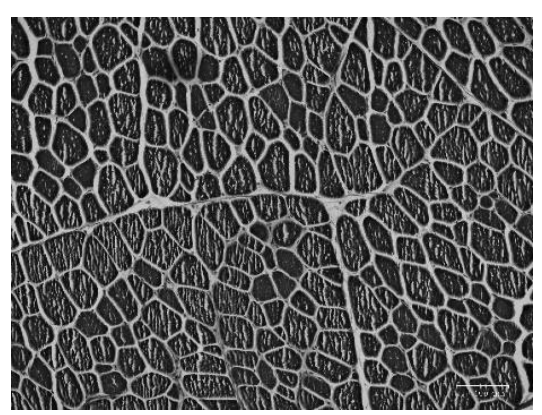

(B)

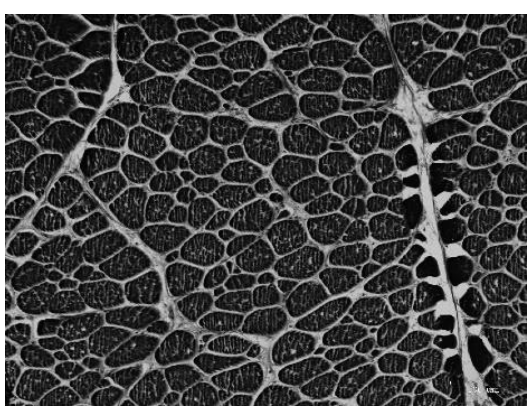

(C)

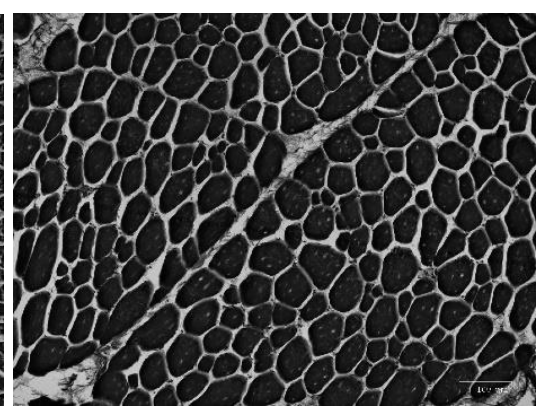

406 Figure 1. Histological cross-section of Longissimus dorsi muscles in piglets. (A) No soil; (B)

407 Antibictorial soil; (C) Normal soil. Bar on lower right corner of each panel $=100 \mu \mathrm{m}$. 


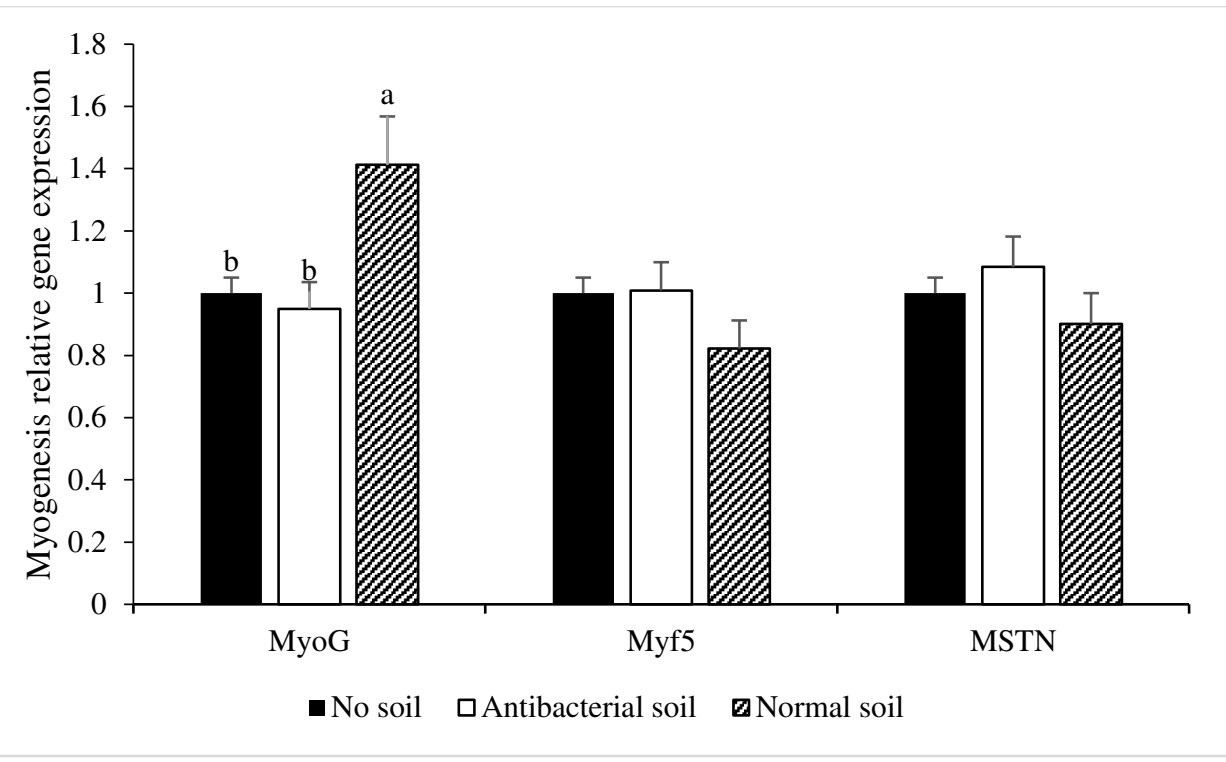

410 Figure 2. Effect of topsoil on gene expression related with myogenesis of weaned piglets. Values

411 are the least square mean \pm standard error of the mean. Within a row, means with no superscripts 412 or with a common superscript letter are not significantly different $(P<0.05)$. 


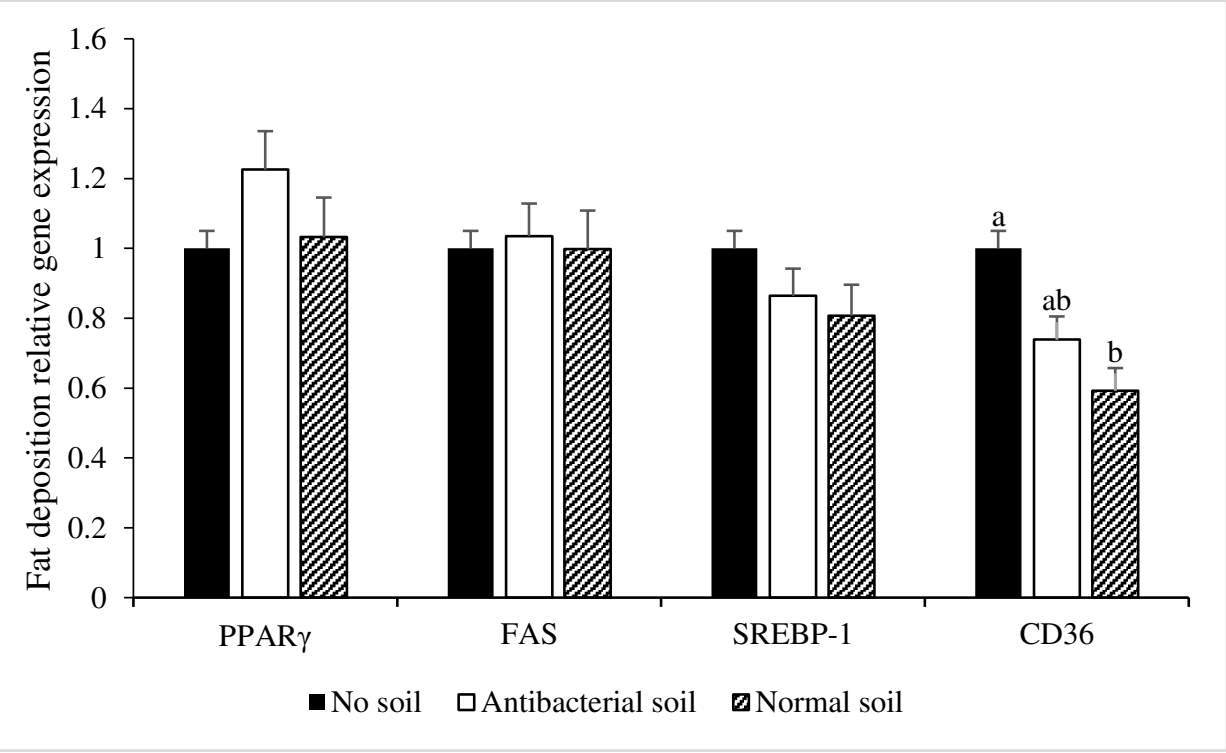

416 Figure 3. Effect of topsoil on gene expression related with intramuscular fat deposition in

417 weaned piglets. Values are the least square mean \pm standard error of the mean. Within a row,

418 means with no superscripts or with a common superscript letter are not significantly different $(P$ $419<0.05)$. 


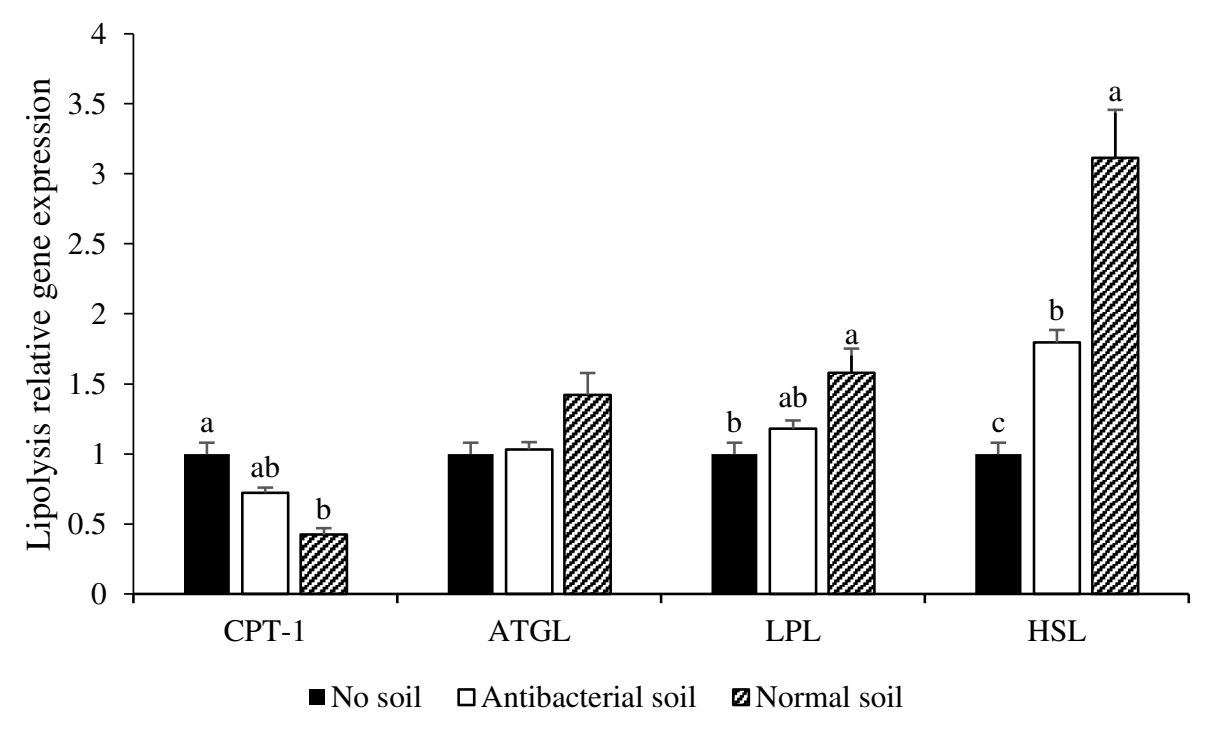

423 Figure 4. Effect of topsoil on gene expression related with intramuscular fat lipolysis in weaned

424 piglets. Values are the least square mean \pm standard error of the mean. Within a row, means with 425 no superscripts or with a common superscript letter are not significantly different $(P<0.05)$. 


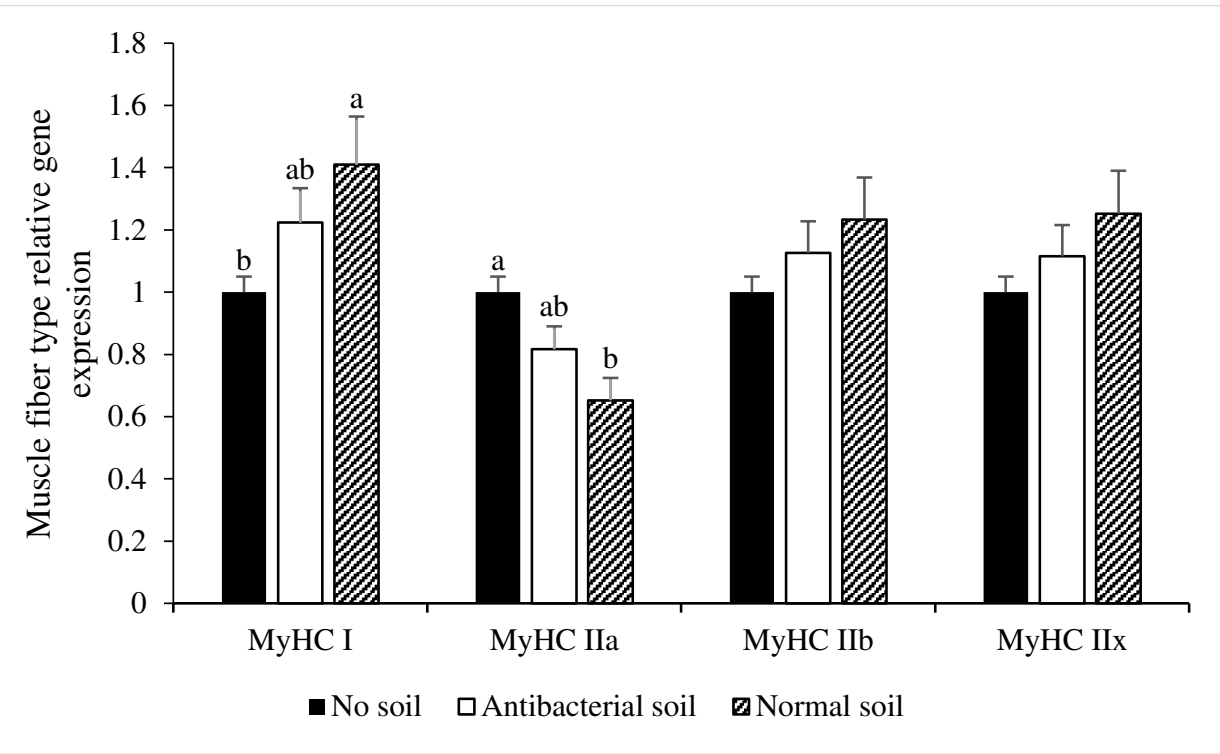

428 Figure 5. Effect of topsoil on gene expression related with muscle fiber type of weaned piglets.

429 Values are the least square mean \pm standard error of the mean. Within a row, means with no

430 superscripts or with a common superscript letter are not significantly different $(P<0.05)$. 

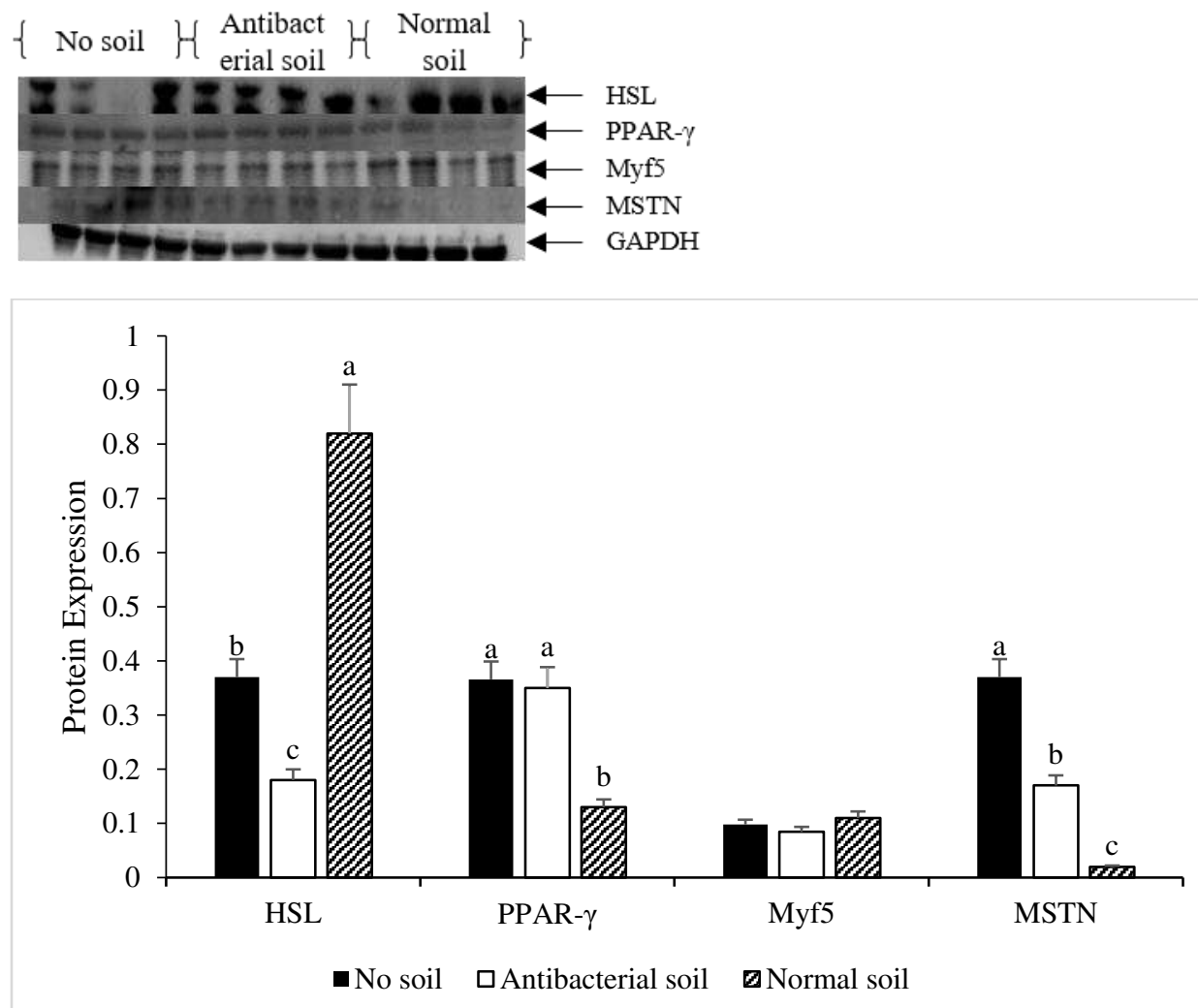

434 Figure 6. Effect of topsoil on protein expression related with intramuscular fat and myogenesis

435 of weaned piglets. Values are the least square mean \pm standard error of the mean. Within a row,

436 means with no superscripts or with a common superscript letter are not significantly different $(P$ $437<0.05)$. 

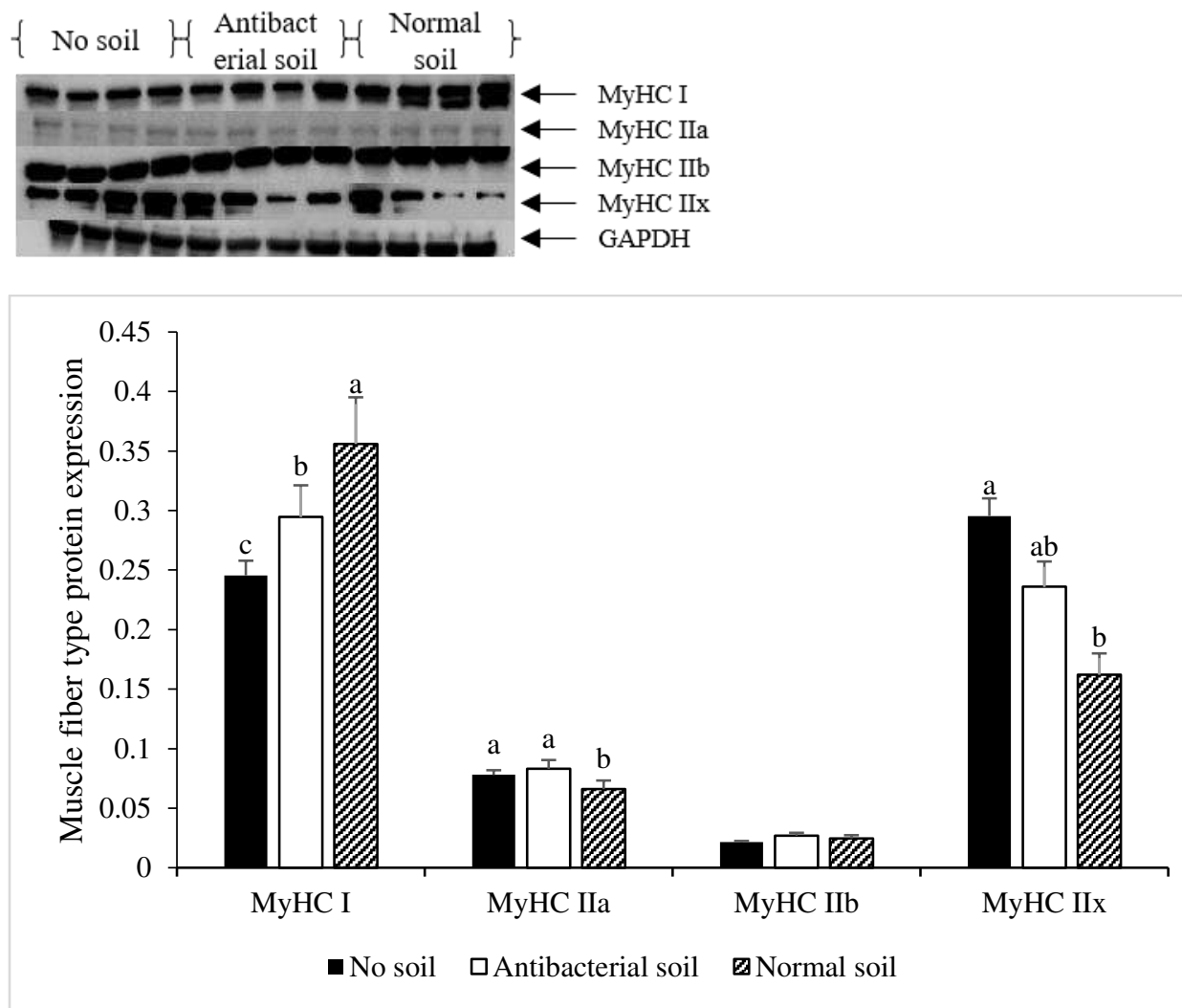

441 Figure 7. Effect of topsoil on muscle fiber type protein expression of weaned piglets. Values are

442 the least square mean \pm standard error of the mean. Within a row, means with no superscripts or 443 with a common superscript letter are not significantly different $(P<0.05)$. 

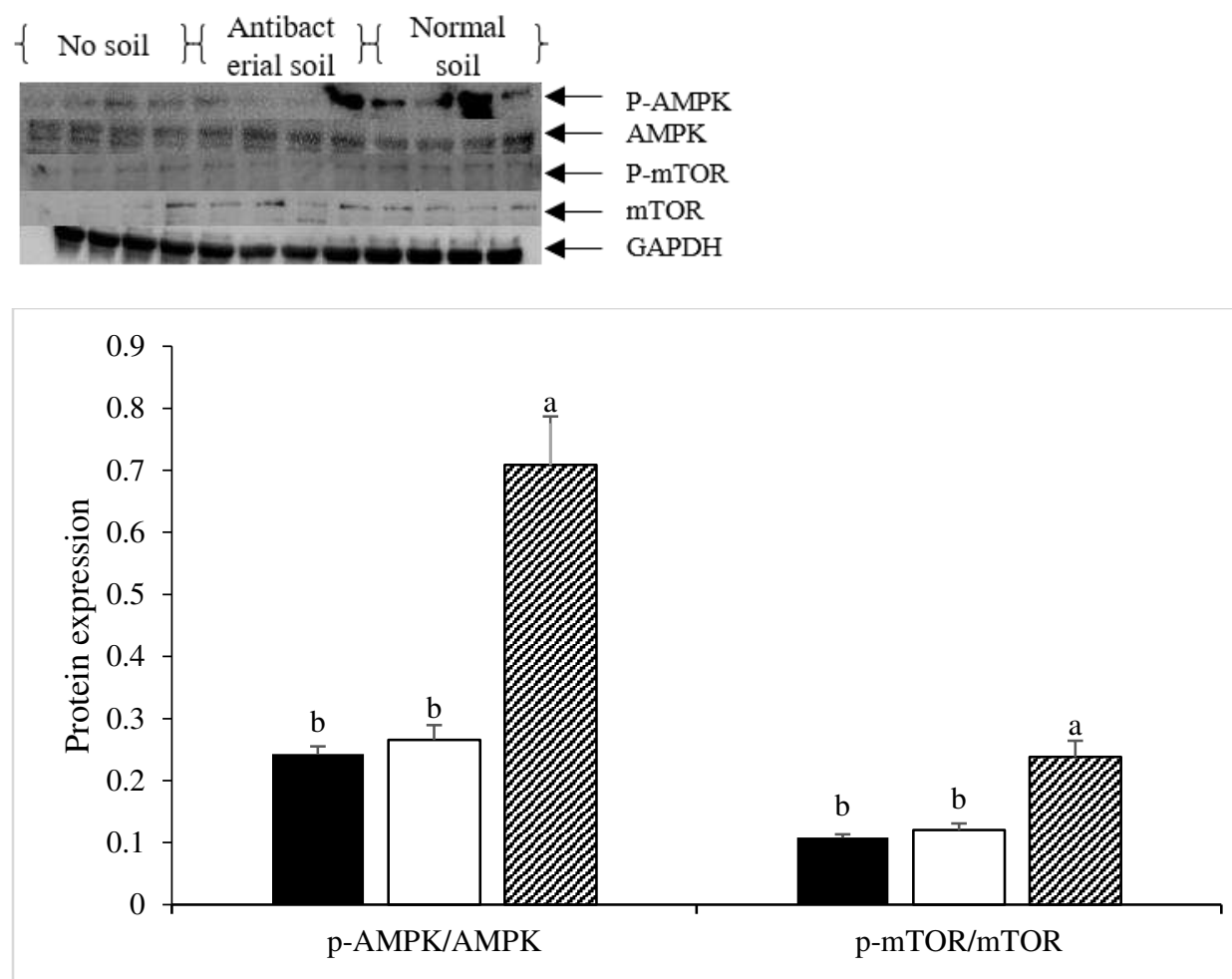

- No soil $\quad$ Antibacterial soil $\quad$ Normal soil

447 Figure 8. Effect of topsoil on AMPK and mTOR signaling pathways in the muscle of weaned

448 piglets. Values are the least square mean \pm standard error of the mean. AMPK: AMP-activated

449 protein kinase; mTOR: mammalian target of rapamycin. Within a row, means with no

450 superscripts or with a common superscript letter are not significantly different $(P<0.05)$. 
Table 1. Primers used in this study

\begin{tabular}{|c|c|c|c|}
\hline Gene $^{1}$ & Direction & Primer sequence & $\begin{array}{c}\text { GenBank } \\
\text { Accession No. }\end{array}$ \\
\hline$\beta$-actin & $\begin{array}{l}\text { Forward } \\
\text { Reverse }\end{array}$ & $\begin{array}{l}\text { 5'- GGATGCAGAAGGAGATCACG -3' } \\
\text { 5'- ATCTGCTGGAAGGTGGACAG -3' }\end{array}$ & DQ845171 \\
\hline MyoG & $\begin{array}{l}\text { Forward } \\
\text { Reverse }\end{array}$ & $\begin{array}{l}\text { 5'- TCT ATG ACG GGG AAA ACT AC -3' } \\
\text { 5'- TGG AGC CAG AGT GGT GTA TC -3' }\end{array}$ & NM_001012406 \\
\hline Myf5 & $\begin{array}{l}\text { Forward } \\
\text { Reverse }\end{array}$ & $\begin{array}{l}\text { 5'-GAGGATATTTCCAGTAAGTGGT -3' } \\
\text { 5'- AGCACTGCTAGCTTTCTCGG -3' }\end{array}$ & NM_001278775 \\
\hline MSTN & $\begin{array}{l}\text { Forward } \\
\text { Reverse }\end{array}$ & $\begin{array}{l}\text { 5' - CTCCTGCTTGACACCGACTT -3' } \\
\text { 5'- TGGGTAGCATGGGGACAGTA -3' }\end{array}$ & NM_214435 \\
\hline PPAR $-\gamma$ & $\begin{array}{l}\text { Forward } \\
\text { Reverse }\end{array}$ & $\begin{array}{l}\text { 5' - ACTCAAAGCAGCAGGAAAGGT -3' } \\
\text { 5'- TGTCACAAACTCACCTTAGGCT -3' }\end{array}$ & NM_214379 \\
\hline FAS & $\begin{array}{l}\text { Forward } \\
\text { Reverse }\end{array}$ & $\begin{array}{l}\text { 5' - CACACTCCTGATCCGCACC -3' } \\
\text { 5'- ACTCCCCATCACAGGGCTAT -3' }\end{array}$ & NM_213839 \\
\hline SREBP-1 & $\begin{array}{l}\text { Forward } \\
\text { Reverse }\end{array}$ & $\begin{array}{l}\text { 5' - TGCAGATAACACAAGCCGGT -3' } \\
\text { 5'- TGCTGCCCGAGAGAAAAGAG -3' }\end{array}$ & AY338729 \\
\hline CD36 & $\begin{array}{l}\text { Forward } \\
\text { Reverse }\end{array}$ & $\begin{array}{l}\text { 5' - GCCACTCCAAGGAGAACAGA -3' } \\
\text { 5'- GCAGGCCACAGTCTTCTACC -3' }\end{array}$ & NM_001044622 \\
\hline CPT-1 & $\begin{array}{l}\text { Forward } \\
\text { Reverse }\end{array}$ & $\begin{array}{l}\text { 5' - CGGAAACGCCTTTTGGACAC -3' } \\
\text { 5'- GACTGGCGGAGGGAATACAG -3' }\end{array}$ & AF284832 \\
\hline ATGL & $\begin{array}{l}\text { Forward } \\
\text { Reverse }\end{array}$ & $\begin{array}{l}\text { 5' - CAAGACTCTGGGTAGCTGCG -3' } \\
\text { 5'- СTTTTCCCCCAGGACTCCAC -3' }\end{array}$ & EU373817 \\
\hline LPL & $\begin{array}{l}\text { Forward } \\
\text { Reverse }\end{array}$ & $\begin{array}{l}\text { 5'- CCAACGTGTCTGTTGTGGAT -3' } \\
\text { 5'- CTGCTTCACCACCTTCTTGA -3' }\end{array}$ & NM_214286 \\
\hline HSL & $\begin{array}{l}\text { Forward } \\
\text { Reverse }\end{array}$ & $\begin{array}{l}\text { 5' - CCAACGTGTCTGTTGTGGAT -3' } \\
\text { 5'- CTGCTTCACCACCTTCTTGA -3' }\end{array}$ & AY559451 \\
\hline MyHC-I & Forward & 5'- CCAACGTGTCTGTTGTGGAT -3' & NM_213855 \\
\hline
\end{tabular}




\begin{tabular}{|c|c|c|c|}
\hline & Reverse & 5'-CTGCTTCACCACCTTCTTGA -3' & \\
\hline \multirow{2}{*}{ MyHC-IIa } & Forward & 5'-CCAACGTGTCTGTTGTGGAT -3' & NM_214136 \\
& Reverse & 5'-CTGCTTCACCACCTTCTTGA -3' & \\
\hline \multirow{2}{*}{ MyHC-IIb } & Forward & 5'-CCAACGTGTCTGTTGTGGAT -3' & NM_001123141 \\
& Reverse & 5'-CTGCTTCACCACCTTCTTGA -3' & \\
\hline \multirow{2}{*}{ MyHC-IIx } & Forward & 5'-CCAACGTGTCTGTTGTGGAT -3' & NM_001104951 \\
& Reverse & 5'-CTGCTTCACCACCTTCTTGA -3' & \\
\hline
\end{tabular}

453 MyoG: myogenin; Myf5: myogenic factor 5; MSTN: myostatin; PPAR- $\gamma$ : peroxisome

454 proliferator activated receptor gamma; FAS: Fas cell surface death receptor; SREBP-1: sterol

455 regulatory element binding transcription factor 1; CD36: cluster of differentiation molecule;

456 CPT-1: carnitine palmitoyltransferase 1; ATGL: adipose triglyceride lipase; LPL: lipoprotein

457 lipase; HSL: hormone-sensitive lipase; MyHC-I: myosin heavy chain I; MyHC-IIa: myosin

458 heavy chain IIa; MyHC-IIb: myosin heavy chain IIb; MyHC-IIx: myosin heavy chain IIx 
460 Table 2. Effect of topsoil on muscle fiber characteristics of weaned piglets

\begin{tabular}{lcccc}
\hline Item & No soil & Antibacterial soil & Normal soil & $P$-value \\
\hline Muscle fiber diameter $/ \mu \mathrm{m}$ & $45.02 \pm 0.53^{\mathrm{c}}$ & $50.53 \pm 0.69^{\mathrm{a}}$ & $48.75 \pm 0.66^{\mathrm{b}}$ & 0.047 \\
Muscle fiber area $/ \mu \mathrm{m}^{2}$ & $1703.24 \pm 41.68^{\mathrm{b}}$ & $2188.73 \pm 61.82^{\mathrm{a}}$ & $2037.1 \pm 58.42^{\mathrm{a}}$ & 0.043 \\
Muscle fiber number & $229.91 \pm 7.69$ & $210.02 \pm 8.04$ & $216.75 \pm 12.62$ & 0.197
\end{tabular}

461 Values are the least square mean \pm standard error of the mean. Within a row, means with no superscripts 462 or with a common superscript letter are not significantly different $(P<0.05)$. 


\section{Supplementary Files}

This is a list of supplementary files associated with this preprint. Click to download.

- SRSupplementaryfile.pdf 